\title{
Spatially and temporally resolved measurements of runaway electrons in the TEXTOR tokamak
}

\author{
T. Kudyakov ${ }^{1}$, K.H. Finken ${ }^{2}$, M.W. Jakubowski ${ }^{3}$, \\ M. Lehnen ${ }^{2}$, Y. Xu ${ }^{4}$, B. Schweer ${ }^{2}$, T. Toncian ${ }^{1}$, \\ G. Van Wassenhove ${ }^{4}$, O. Willi ${ }^{1}$ and the TEXTOR team ${ }^{2}$ \\ ${ }^{1}$ Institut für Laser und Plasmaphysik, Heinrich-Heine-Universität Düsseldorf, \\ D-40225 Düsseldorf, Germany \\ ${ }^{2}$ Institut fuer Energieforschung - Plasmaphysik, Forschungszentrum Juelich \\ GmbH, Association EURATOM-FZJ, Trilateral Euregio Cluster, D-52425 \\ Juelich, Germany \\ ${ }^{3}$ Max-Planck-Institut für Plasmaphysik, IPP-EURATOM Association, D-17491 \\ Greifswald, Germany \\ ${ }^{4}$ Laboratoire de Physique des Plasmas/Laboratorium voor Plasmafysica, \\ ERM/KMS, EURATOM Association, B-1000 Brussels, Belgium \\ E-mail: Timur.Kudyakov@uni-duesseldorf.de
}

\begin{abstract}
Spatially and temporally resolved measurements of runaway electrons have been performed in the TEXTOR tokamak. The main diagnostic for the investigations is a newly developed runaway probe which is mounted at a reciprocating mechanism. In addition, the runaway electrons are studied in the plasma by synchrotron emission. The scanning probes consists of fluorescing crystals covered by a graphite housing and is located at different radial positions. In this study, two generations of the probes are presented, a simple one for proving the principle and a more advanced one which allows the measurement of the energy distribution of the runaways. A strong decay of the runaway electrons at the plasma edge was observed. Runaway electrons have been measured at different plasma densities by synchrotron radiation and the probe. The reduction of the number of runaways have been measured with increasing plasma densities during low density plasma discharges by both diagnostics. The spectrum of runaway electrons produced during low density plasma discharge was measured. Finally, measurements of runaways with high temporal resolution of $0.05 \mathrm{~ms}$, have been carried out during a disruption discharge.
\end{abstract}

PACS numbers: 52.55.Fa, 52.35.Py, 52.40.Hf 


\section{Introduction}

In tokamaks, runaway electrons with energies in the $\mathrm{MeV}$ range are typically generated in low density discharges or under conditions of an enhanced loop voltage (plasma start up $[1,2,3]$, disruptions $[4,5,6,7]$ or instabilities $[8,9])$. The runaway generation during disruptions is a possible risk for the integrity of the device. The risk increases with the size of the tokamak; estimates have shown that up to $700 \mathrm{MJ}$ of energy can be transferred by the runaway electrons in International Thermonuclear Experimental Reactor (ITER) to the wall [10]. These electrons can easily penetrate low Z plasma facing materials (carbon, beryllium) and damage high Z structural walls $[10,11,12]$. Because of their large energy, the runaway electrons can be regarded as effectively collisionless. Aside from the fusion, electrons with energies in $\mathrm{MeV}$ range are also interesting in the study of novel mechanisms for electric breakdown in gases [13] and in astrophysical plasmas [14].

The mechanisms responsible for the generation of runaway electrons in the plasma current are described in refs $[15,16,17,18,19,20,21,22,23]$. In a primary generation process $[15,16,17,18,19]$ electrons gain energy from the electric field and lose energy through Coulomb collisions. An electron with velocity equal or larger than the critical velocity, for which the collisional drag force balances the accelerating electric field, becomes a runaway electron. A secondary generation mechanism [3, 20, 21, 22, 23] can be important for runaway generation if runaways of sufficient energy are already present. In the secondary process, the runaway electrons are generated due to the interaction of the runaway electrons with thermal electrons.

Runaway electrons have been studied at the vessel wall or in the plasma core but no measurements have so far been carried out at the plasma boundary. At the vessel wall several techniques have been used. For example, hard x-ray were observed during thick-target interactions with runaway electrons [24, 25]. Further, neutrons produced in $(\gamma, n)$ photo-nuclear reactions due to interactions of runaway electrons with the plasma facing materials $[25,26]$ were measured. Measurements of hard x-ray radiation produced when runaway electrons impinged on the wall [25] were carried out. On the other hand, information about runaway electrons inside the plasma was obtained by synchrotron radiation in the mid IR spectral range $(4-8 \mu \mathrm{m})$, produced by the highly relativistic electrons propagating in the toroidal direction [1]. With this diagnostic runaway electrons with energies above $25 \mathrm{MeV}$ were measured inside the plasma.

Somewhat less quantitative is electron cyclotron emission (ECE) measurements because of the competition of the superthermal electron emission and the reabsorption in the optically thick plasma [27]

\section{Experimental set-up}

In this paper comprehensive measurements of runaway electrons performed at the TEXTOR tokamak are reported. TEXTOR is a medium size tokamak and has the following parameters: major radius $R=1.75 \mathrm{~m}$ and minor radius $a=0.46 \mathrm{~m}$. Measurements were performed in deuterium discharges with the following plasma parameters: plasma current $I_{p}=300 \mathrm{kA}$; magnetic field $B_{t}=2.25 \mathrm{~T} ; n_{e}=0.7 \cdot 10^{19} \mathrm{~m}^{-3}$.

Fig. 1 gives an overview about the runaway detectors used on TEXTOR. In 


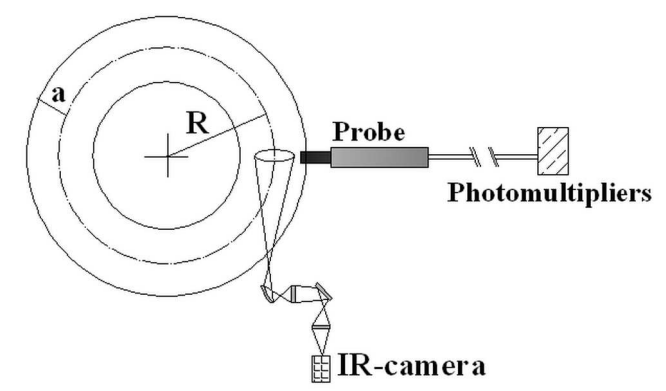

Figure 1. The experimental set-up. Synchrotron radiation can register runaways with energies higher than $25 \mathrm{MeV}$ in the plasma center. A novel probe measures electrons at the plasma edge.

the TEXTOR hall a set of detectors is positioned for recording hard x-rays and neutrons which are created by the impingement of the runaways on the walls. These detectors are originally designed to measure the ion temperature of strongly heated deuterium plasmas but they provide also information of runaway electrons exceeding an energy of a few $\mathrm{MeV}$. The other and rather unique runaway detection system is the synchrotron detection using IR radiation between $3 \mu \mathrm{m}$ and $5 \mu \mathrm{m}$ which is oriented in electron approach direction. The IR-camera, recording the synchrotron radiation, yields spatially and temporally $(1 \mathrm{kHz})$ resolved measurements of runaway electrons with energies above $25 \mathrm{MeV}$ between $1.4 \mathrm{~m}$ and $1.9 \mathrm{~m}$ of the major radius. The synchrotron radiation is emitted in tangential direction to the magnetic field curvature with divergence angle $\theta=(8-9) \times 10^{-2}$. More detailed description of the synchrotron radiation technique can be found in [1]. A runaway probe mounted on a reciprocating mechanism is used as the main diagnostic in the presented contribution.

The probe consists of a set of scintillating YSO $\left(\mathrm{Y}_{2} \mathrm{SiO}_{5}: \mathrm{Ce}\right)$ crystals $\dagger[28]$ which are connected via fiber optics to photomultipliers (Hamamatsu R3896). The probe is located in the equatorial midplane of TEXTOR at the low field side. The light output of the scintillators is a function of the runaway energy and is proportional to the number of incident runaway electrons. The main aim of the probe is the development of a tool which provides information of the energy distribution of the runaways. Two probes were tested as seen in Fig. 2. The first one is a rather simple design which shows the principle idea. A scintillator is located behind a $5 \mathrm{~mm}$ thick graphite housing. The second scintillator is separated by a piece of $8 \mathrm{~mm}$ tungsten. The graphite housing shields the detectors from the ambient light and low energy particles. Only runaway electrons with an energy exceeding $2.5 \mathrm{MeV}$ can pass through the graphite†. The tungsten separates runaways in the electron approach direction from those in the ion approach direction (positrons or background signal). However, it turned out that the runaways are scattered on the top plate of the graphite such that the remote scintillator also sees runaways. We make use of the signal of the remote scintillator because the first one became strongly saturated during typical runaway discharges.

$\dagger$ YSO crystals produced by Proteus Inc., (http://www.proteus-pp.com).

$\uparrow$ The calculation of the minimum detected energy of electrons was done using Geant4 software developed by Members of the Geant4 Collaboration (http://cern.ch/geant4). 
The crystals have the following characteristics: melting point of 2470 degree Celsius, length $20 \mathrm{~mm}$, cross section of $1 \mathrm{~mm}^{2}$, decay time and peak emission of $42 \mathrm{~ns}$ and $420 \mathrm{~nm}$ respectively, light output of 10000 photons/MeV [28] or 30000 photons $/ \mathrm{MeV} \dagger$. Different light outputs of the crystal depend on its production technique, in the present experiment crystal with light output 30000 photons/MeV† have been used. The crystal length was chosen in order to have enough signal. For the present purpose, the probe is inserted relatively slowly to the plasma edge with a velocity of $0.5-0.7 \mathrm{~m} / \mathrm{s}$.

The positive results of the first probe encouraged the development of a second probe type which is shown in Fig. 3). The aim is to resolve runaway electrons with energies between 5 and $30 \mathrm{MeV}$ with energy resolution about $3-4 \mathrm{MeV}$. The same type of YSO crystals like in the first probe, but their length was reduced to $2 \mathrm{~mm}$; as this length provides sufficient signal and a good radial resolution. The crystals are shielded by different thicknesses of tungsten filters, ranging from $0 \mathrm{~mm}$ to a maximum of $6 \mathrm{~mm}$, in front of the direction of runaway electrons. From the other side and from the top, the crystals are shielded by tungsten with thicknesses between 6 and $10 \mathrm{~mm}$. Simulations with a Monte-Carlo GEANT4 code $\dagger$ of the probe show that the sensitivity of the crystals to the electrons are two order of magnitude higher than to $\mathrm{x}$-ray radiation in the measured energy range.

During the motion runaway electrons at different minor radial positions are measured. A comparative measurement of the first and the second probe designs are shown in Fig. 4. Here the probe is radially moved during the stationary phase of a runaway discharge such that the ordinate represents the radial position of the probe. Both measurements show a good agreement of the radial profile of the runaways in the scrape off layer. To our knowledge, these are the first measurements in which probes were inserted directly inside the plasma giving the possibility to measure directly runaway electrons at the plasma edge.

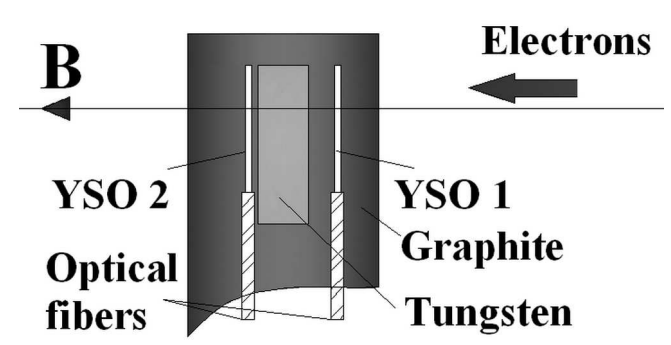

Figure 2. The first probe design, the presented part of the probe demonstrates the position of the YSO crystals inside the graphite housing. The second crystal is shielded by $8 \mathrm{~mm}$ tungsten, which allows to measure only scattered runaway electrons in the graphite housing with a spatial resolution of $2 \mathrm{~cm}$.

Special investigations have been performed whether the runaway signal is disturbed by bremsstrahlung or neutrons $((\gamma, n)$ photo-nuclear reactions) created by the impact of the runaway electrons with the walls. Since this radiation is diffuse inside 
TEXTOR, it should contribute to the signal independent of its position. Fig. 4 which displays the radial signal of the runaways from both probe designs drops clearly to zero at a minor radius of $49 \mathrm{~cm}$. This indicates that this background can be neglected. In addition, discharges were performed with neutron beam injections (NBI) where a big flux of neutrons impinged on the probe. It was found that the YSO crystals are not sensitive to these neutrons. Signals registered by the neutron detectors were one order of magnitude higher for the NBI discharges than for the runaway ones. A more detailed description of the probe and a method of the spectrum analysis can be found in [29].

The new diagnostic has the advantage that - beside the direct measurement of the runaway electrons - the local interaction of runaways with materials can be investigated. The time interval of the measurement and the radial penetration depth of the probe into the plasma are limited by the heat flux of the thermal and of the runaway electrons. The destruction limit of the probe is $2 \mathrm{~cm}$ inside the last closed field surface (LCFS) at $50 \mathrm{~ms}$ exposure time. Simple simulation of the energy loaded on the graphite part of the probe can be made under certain conditions: an active cross section of the probe $S=6 \cdot 10^{-4} \mathrm{~m}^{2}$, thickness $d=0.005 \mathrm{~m}$. Electrons with energy higher than $3 \mathrm{MeV}$ lost about $E_{a b s} \approx 2 \mathrm{MeV}$ in the graphite part. The number of runaway electrons at the plasma edge $n_{r}=10^{14}(1-0.45 / 0.46)^{1.5} \approx 3 \cdot 10^{11} \mathrm{~m}^{-3}$ in the TEXTOR tokamak [2], thus energy deposited on the graphite part during $\Delta t=50 \mathrm{~ms}$ can be estimated as, $E_{\text {dep }}=n_{r} S c \triangle t E_{a b s} \approx 1 \mathrm{~kJ}$, which already can cause some damages of the graphite housing. The energy load on the graphite housing for the same plasma conditions $\left(n_{e}=0.7 \cdot 10^{19} \mathrm{~m}^{-3}\right)$, due to the ions and thermal electron transport, is about one order of magnitude less than due to the runaway electron transport. On the other hand, $1 \mathrm{~cm}$ outside of the LCFS the probe can be used during the whole discharge, without damage.

The possible disturbances on the runaway electron flux which can be caused by the probe insertion was neglected due to the following reasons: i) the time duration of the probe insertion is very long in comparison to the time which necessary to a relativistic electron to make one turn around the tokamak, therefore the probe can be regarded as a stationary placed object for the runaway electrons; ii) the probe has relatively small size in compare to the limiter, the insertion of the probe was to $46 \mathrm{~cm}$ which defined by the limiter position. Thus the disturbance produced by the probe can be considered as a local one and its influence can be neglected on the runaway electron dynamics.

\section{Runaway electrons at the plasma edge}

As already mentioned before, first measurements with both probes were performed for the determination of the radial decay of the runaway density at the edge and in the scrape-off layer of the plasma. In the scanning mode regime the insertion of the first probe starts $3.4 \mathrm{~s}$ after the beginning of the discharge with an insertion time of $0.5 \mathrm{~s}$. The probe remains at the innermost position for $0.1 \mathrm{~s}$ and then it is withdrawn within $0.5 \mathrm{~s}$. A signal from the first probe as a function of the crystal position is shown in Fig. 4. The crystal is placed at $45.5 \mathrm{~cm}$ minor radial position. The Last Closed Flux Surface (LCFS) was defined by a carbon limiter and amounts to $46 \mathrm{~cm}$; hence the measurements are carried out up to $0.5 \mathrm{~cm}$ inside the plasma. Similar measurements 


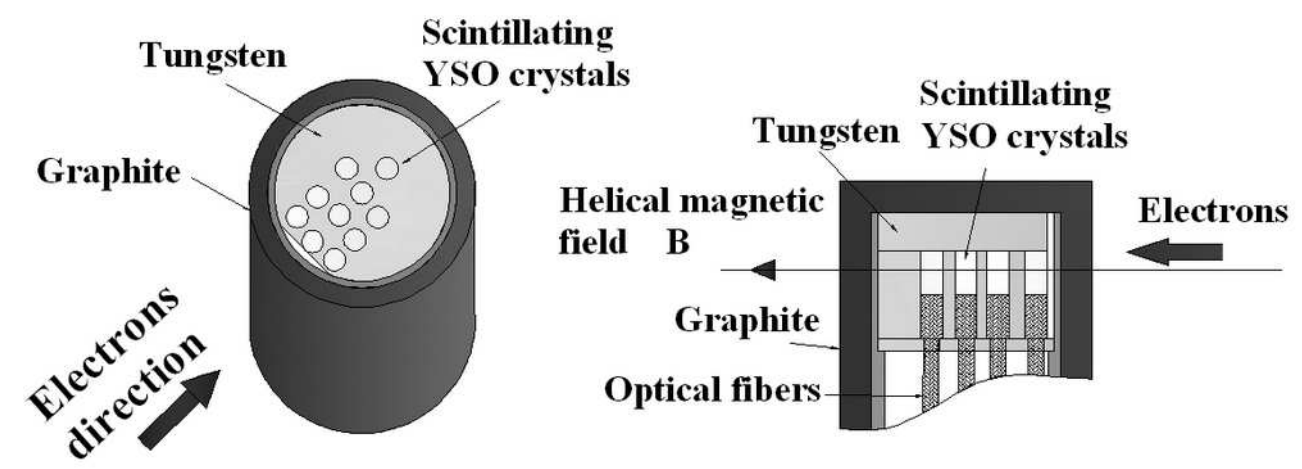

Figure 3. The design of the second probe. The probe consists of ten YSO crystals and has $2 \mathrm{~mm}$ spatial resolution. The light produced in the crystals is transferred to photomultipliers by optical fibers.

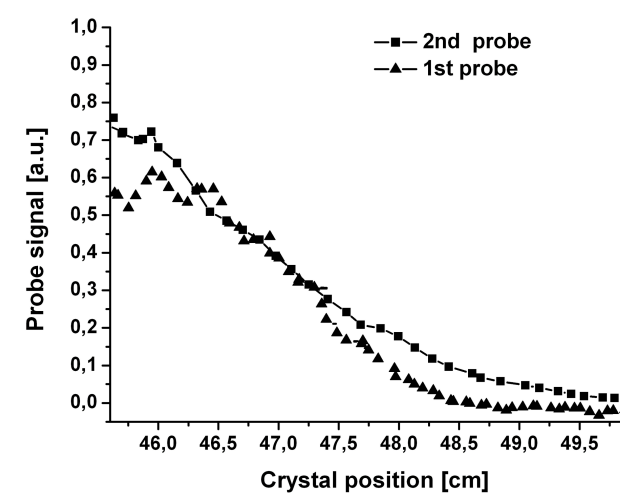

Figure 4. Measurements of runaway electrons between $53 \mathrm{~cm}$ and $45 \mathrm{~cm}$ of the minor radius by the both scanning probe. Electrons with energies $E>2.5 \mathrm{MeV}$ were registered by the first probe. In the second probe signal from the first crystal is presented, electrons with energies, $E>5 \mathrm{MeV}$, were registered. A limiter position is at $46 \mathrm{~cm}$.

of runaway electrons by using the second probe have been performed. The probe is inserted at $3.1 \mathrm{~s}$ after the beginning of the discharge with velocity $0.7 \mathrm{~s}$ into the plasma with crystals position at $45.5 \mathrm{~cm}$. The signals in Fig. 4 from the first crystal show the electrons with energies higher than $5 \mathrm{MeV}$. The steep rise of the signal demonstrates a rapid increase of the runaway electron population starting at the $48.5 \mathrm{~cm}$ minor radial position. In spite of different sensitivities and spatial resolutions of the probes, a good correlation between both measurements can be observed. Runaway scans have been performed by the second probe for a set of discharges with slightly different densities between $0.6 \cdot 10^{19} \mathrm{~m}^{-3}$ and $1.2 \cdot 10^{19} \mathrm{~m}^{-3}$; the high reproducibility has been observed.

Open field lines at minor radial positions higher than $46 \mathrm{~cm}$ can be responsible for the strong runaway loss that causes the exponential decay of the runaway electron populations between $46 \mathrm{~cm}$ and $48.5 \mathrm{~cm}$. 


\section{Production of runaways at different densities}

It is well known that the runaway production according to the Dreicer model depends strongly on the electron density. Therefore a set of discharges was performed in which the electron density was systematically increased from discharge to discharge; during the discharge, the density was kept constant. The runaway density was measured at $2.1 \mathrm{~s}$ after the beginning of the discharge by using synchrotron radiation and probe measurements of runaways. In this series probe \#2 was used which allows a discrimination of the runaway energy. Fig. 5a) and b) show the synchrotron data together with time traces of the probe channels for energies higher than 8; 16 and $25 \mathrm{MeV}$. The number of runaways reduces by one order of magnitude when the density grows by a factor of two. The synchrotron radiation is in good agreement with the probe signals; perhaps it falls slightly stronger than for the probe channel measured in the same energy range of electrons.

The reduction of the number of runaways with increasing densities can be

a)

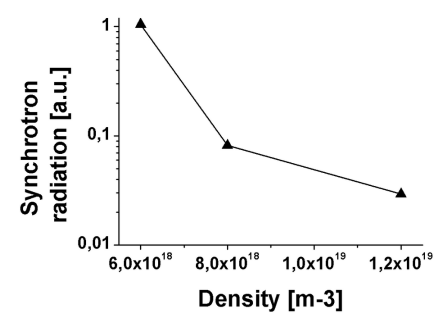

b)

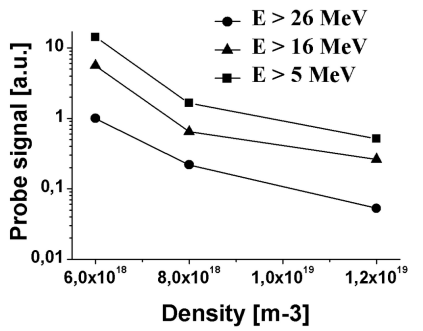

c)

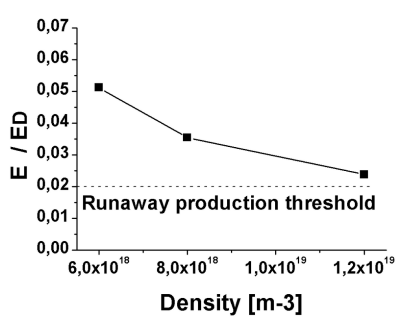

Figure 5. a) Synchrotron radiation, electrons with energies higher than $25 \mathrm{MeV}$ at $2.15 \mathrm{~s}$ into the discharge; b) The probe measurements of runaway electrons with electron energies higher than $5 \mathrm{MeV}, 16 \mathrm{MeV}$ and $26 \mathrm{MeV}$. The probe was placed at the last closed flux surface (LCFS) at $2.15 \mathrm{~s}$ during the discharge. c) Ratio of the electric field to the Dreicer field.

explained by the suppression of primary generated electrons. The production rate of primary generated electrons is an exponential function on $E_{c} / E_{\|}[16,19,5]$ and is given as:

$$
\dot{n}_{r}^{p r}=\frac{n_{e}}{\tau}\left(\frac{m_{e} c^{2}}{2 T_{e}}\right)^{3 / 2}\left(\frac{E_{c}}{E_{\|}}\right)^{3 / 8} \exp \left(-\frac{E_{c}}{4 E_{\|}}-\sqrt{\frac{2 E_{c}}{E_{\|}}}\right),
$$

where $\tau=4 \pi \epsilon_{0}^{2} m_{e}^{2} c^{3} / n_{e} e^{4} \ln \Lambda$ is the relativistic electron collision time, $E_{\|}$is the parallel electric field and $E_{c}=n_{e} e^{3} \ln \Lambda / 4 \pi \epsilon_{0}^{2} m_{e} c^{2}$ the critical electric field. Hence the increasing density causes the increase of $E_{c}$ and as a consequence the reduction of $\dot{n}_{r}^{p r}$. Although the production rate of secondary generated electrons does not depend explicitly on the plasma density $[23,5]$, nevertheless the reduction of $\dot{n}_{r}^{p r}$ leads to a decrease of $n_{r}$ and the whole suppression of the runaway electron production, as seen in:

$$
\dot{n}_{r}^{s e c} \simeq n_{r}\left(\frac{\pi}{2}\right)^{1 / 2} \frac{E_{\|} / E_{c}-1}{3 \tau \ln \Lambda} .
$$

Estimates of the ratio of the electric field to the Dreicer field, $E_{D}=m_{e} c^{2} E_{c} / T_{e}$, for corresponding densities, with the following other parameters: $\ln \Lambda=16, T_{e}=1.5 \mathrm{keV}$ [3], are shown in Fig. 5c. At $n_{e}=1.2 \cdot 10^{19} \mathrm{~m}^{-3}$ the value $E_{\|} / E_{D}=0.25$ which 
corresponds to the threshold of the runaway electron production [5] below which almost no runaway production occurs. At this value the number of runaways registered by both diagnostics was close to zero and one order of magnitude smaller than at $n_{e}=0.7 \cdot 10^{19} \mathrm{~m}^{-3}$.

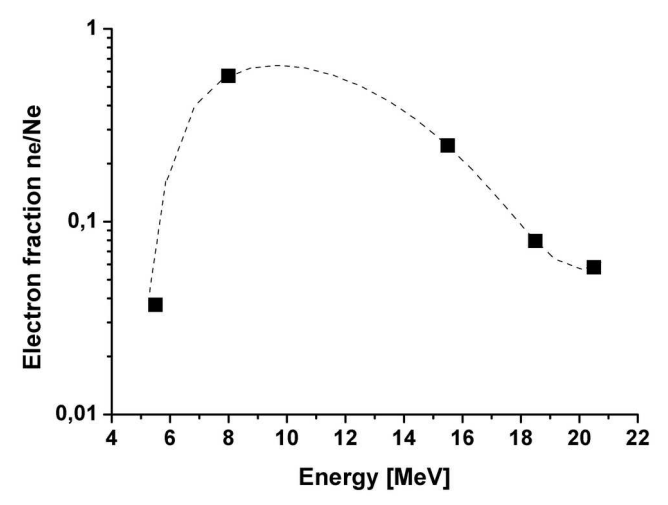

Figure 6. Runaway electron spectrum measured during the low density plasma discharge, $n_{e}=0.8 \cdot 10^{19} \mathrm{~m}^{-3}$. The probe was inserted to the Last Closed Field Surface at $2.15 \mathrm{~s}$ into the discharge.

The main advantage of the probe \#2 is the possibility to provide information of the spectrum of runaway electrons with energies between 5 and $30 \mathrm{MeV}$. This is in so far of high interest as a) the spectrum has never been measured and b) one expects differences in the spectrum between primary generated runaways (Dreicer effect) and secondary generated ones. It is expected that the secondary generated electrons play the dominant role during disruptions. The analysis of the probe signal of the middle point in Fig. 5 b) provides the spectrum shown in Fig. 6; the spectrum of the runaway electrons was measured at $2.15 \mathrm{~s}$ during a low density plasma discharge, with a density $n_{e}$ of $0.8 \cdot 10^{19} \mathrm{~m}^{-3}$. The analysis of the spectrum has been performed by comparing signals from the first five crystals of the probe [29]. The measurement shows that most of runaways have energies between 6 and $16 \mathrm{MeV}$. After $1 \mathrm{~s}$ of the beginning of the discharge, runaway electrons are mostly produced due to the secondary generation process [3] and the cross section for the close collisions is higher for electrons with energies of about $8 \mathrm{MeV}$ than for the ones with $25 \mathrm{MeV}$. Additionally, during close collisions, a runaway electron transmits a part of its energy to another thermal one, resulting in a redistribution of the spectrum.

\section{Time dependent events (disruptions)}

The probe can measure electrons with the temporal resolution of about $200 \mathrm{~ns}$ and the data acquisition is mostly limited by a data logger memory capacitor which equals about $0.05 \mathrm{~ms}$. The high temporal resolution of the probe allows to study runaway electrons produced during a plasma disruptions. In Fig. 7 the first measurements of runaway electrons during plasma disruptions by the scanning probe are shown. Fig. 7a shows the time trace of the plasma current during the disruption. The disruption is 

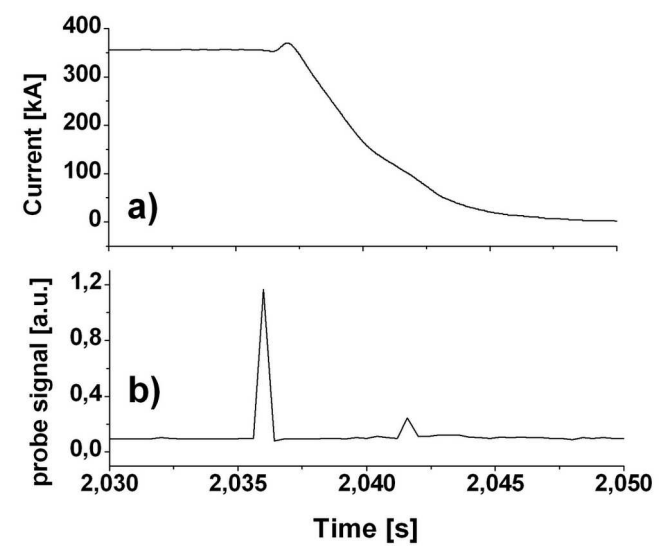

Figure 7. a) Plasma current, plateau formation at $2.04 \mathrm{~s}$; b) The probe measurements of runaway electrons with electron energies higher than $8 \mathrm{MeV}$. The probe was placed at the minor radial position of $47 \mathrm{~cm}$.

typically initiated by a negative voltage spike and a transient increase of the plasma current due to the lowering of the plasma inductance during the energy quench [30]. The current quench shows a small plateau during which runaway electrons are created.

Fig. 7b shows the probe signal. Here one can see two peaks, a larger one during the energy quench and a smaller one after the plateau phase of the current quench. We interpret first peak as the loss of runaways which were created during the startup phase of the discharge $[31,32]$ and the second peak generated during the current quench phase [30].

\section{Conclusions and Outlook}

In conclusion, measurements of runaway electrons have been performed by two generations of runaway probes. The first generation is a relatively simple design which acts as a proof of principle. After its successful application, a second probe was developed which provides time resolved information of the energy distribution of the runaways. The measurements are complemented by synchrotron radiation yielding information over the whole area of plasma. The new diagnostic was used to measure directly runaway electrons near the plasma boundary of a Tokamak plasma for the first time. The interesting point is that the diagnostic fills a gap by measuring profiles at the plasma edge and that it directly measures the runaway electrons. In combination with measurements of the synchrotron radiation the runaway electrons were studied in the interior and exterior region of the plasma. Measurements of the runaway electrons at the plasma boundary show that the population of the runaway electrons strongly increase up to the last closed flux surface. An analysis of runaway production at different densities by the synchrotron radiation and the scanning probe shows that the number of runaway electrons decreases with increasing density and the production of runaways is completely suppressed at $E_{\|} / E_{D}=0.25$. The main advantage of the probe diagnostic is its possibility to measure the runaway electron spectrum over a 
wide energy range with the high temporal resolution. This characteristic makes the probe a very promising diagnostic to study runaway electrons produced during plasma disruptions.

\section{Acknowledgments}

The authors acknowledge fruitful discussions with Prof. Dr. Spatschek, the assistance of the staff at TEXTOR and A. Hiller. Funding of this work by GRK1203 and F and E contract and "Partners in the Trilateral Euregio Cluster (TEC)" is acknowledged.

\section{References}

[1] K.H. Finken, J.G. Watkins, D. Rusbuldt, W.J. Corbett, K.H. Dippel, D.M. Goebel, R.A. Moyer, Nucl. Fus. 30, 859 (1990).

[2] R. Jaspers, "Relativistic runaway electrons in tokamak plasmas" Ph.D Thesis, Eindhoven University of Technology, The Netherlands (1995).

[3] R. Jaspers, K.H. Finken, G. Mank, F. Hoenen, J.A. Boedo, N.J.L. Cardozo and F.C. Schuller, Nucl. Fus. 33, 1775 (1993).

[4] R.D. Gill, Nucl. Fus. 33, 1613 (1993)

[5] P. Helander, L.-G. Eriksson, and F. Andersson, Plas. Phys. Contr. Fus. 44, B247 (2002).

[6] L.-G. Eriksson, P. Helander, F. Andersson, D. Anderson, and M. Lisak, Phys. Rev. Lett. 92, 205004 (2004).

[7] V.V. Plyusnin, V. Riccardo, R. Jaspers, B. Alper, V.G. Kiptily, J. Mlynar, S. Popovichev, E. de La Luna F. Anderson et.al., Nucl. Fus. 46, 277 (2006).

[8] V.V. Parail, O.P. Pogutse, Nucl. Fus. 18, 303 (1978).

[9] P.V. Savrukhin, Phys. Rev. Lett. 86, 3036 (2001).

[10] S. Putvinski, P. Barabaschi, N. Fujisawa, N. Putvinskaya, M.N. Rosenbluth, J. Wesley, Plasma Phys. Control. Fusion 39 B157 (1997).

[11] 1999 ITER physics basis, Nucl. Fus. 39, 2137 (1999).

[12] H. Knoepfel and D.A. Spong, Nucl. Fus. 19, 785 (1979).

[13] A.V. Gurevich, G.A. Milikh and R. Roussel-Dupre, Phys. Lett. A 165, 463 (1992).

[14] A. V. Gurevich and R. N. Sudan, Phys. Rev. Lett. 72, 645 (1994)

[15] H. Dreicer, Phys. Rev. 115, 238 (1959).

[16] A.V. Gurevich, Sov. Phys. JETP 12, 904 (1961).

[17] M.D. Kruskal and I.B. Bernstein, PPPL Report MATT-Q-20, p174 (1962)

[18] R.H. Cohen, Phys. Fluids 19, 239 (1976).

[19] J.W. Connor and R.J. Hastie, Nucl. Fus. 15, 415 (1975).

[20] Yu.A. Sokolov, JETP Lett. 29, 218 (1979).

[21] N.T. Besedin and I.M. Pankratov, Nucl. Fus. 26, 807 (1986).

[22] R. Jayakumar, H. H. Fleischmann, and S. J. Zweben, Phys. Lett. A 172, 447 (1993).

[23] M.N. Rosenbluth and S.V. Putvinski, Nucl. Fus. 37, 1355 (1997).

[24] B. Esposito, R.M. Solis, P. vanBelle, O.N. Jarvis, F.B. Marcus, G. Sadler, R. Sanchez, B. Fischer, P. Froissard, J.M. Adams, E. Cecil, N. Watkins, Plas. Phys. Contr. Fus. 38, 2035 (1996).

[25] B. Esposito, J.R. Martin-Solis, R.M. Poli, J.A. Mier, R. Sanchez, L. Panaccione, Phys. Plas. 10, 2350 (2003).

[26] O.N. Jarvis, G. Sadler, J.L. Thompson, Controlled Fusion and Plasma Heating (Proc. 15th Eur.Conf. Dubrovnik, 1988), Vol. 12B, Part I, European Physical Society 334 (1988).

[27] K.H. Finken, S.S. Abdullaev, M.W. Jakubowski, R. Jaspers, M. Lehnen, R. Schlickeiser, K.H. Spatschek, A. Wingen, R. Wolf, Nucl. Fus. 47, 91 (2007).

[28] M. Balcerzyk, M. Moszynski, M. Kapusta, D. Wolski, J. Pawelke, C.L. Melcher, IEEE Trans. Act. Nucl. Sci. 47, 1319 (2000).

[29] T. Kudyakov, K.H. Finken, M. Jakubowski, M. Lehnen, Y. Xu, O. Willi, Rev. Scient. Instrum. 79 (2008, in print).

[30] M. Lehnen, S.A. Bozhenkov, S.S. Abdullaev and M. W. Jakubowski, Phys. Rev. Lett. 100 255003 (2008). 
[31] G. Fussmann, Nucl. Fus. 19, 327 (1979).

[32] B. Kurzan, K. -H. Steuer, and G. Fussmann, Phys. Rev. Lett. 75, 4626 (1995). 